\title{
Face Gesture Pattern Recognition using Muscle Distributed Sensor for Face off Recovery
}

\section{Yothapakdee $\mathrm{K}^{1}$ and Yupapin $\mathrm{P}^{2 *}$}

${ }^{1}$ Department of Computer Science, Faculty of Science and Technology, Loei Rajabhat University, Thailand

${ }^{2}$ Faculty of Electrical and Electronics Engineering, Ton Duc Thang University, Ho Chi Minh City, Vietnam

In the past, human used to think that many things in this world were impossible. However, rapid advances in modern technology as seen nowadays are being witnessed by the public [1]. In particular, the development of science and medicine has a direct impact on lives of human beings. Consequently, it would encourage people to live longer [2]. As can be seen from a surgery for an organ transplant being done to provide assistance to patients injured from an accident or a group of people with disabilities, transplant surgery, which generally known as face transplant or face off, can change the face from one to another. Also, a person may want to change some of the organs in the body for enhancing his/her beauty or recovering from a change of time due to the nature of the human body. One of the common forms of cosmetic surgery is intended to enhance facial features with a process of cutting and removing some of the excess facial skin and tighten the remaining skin, which is known as a face-lift [3]. Unfortunately, in many cases, an injury and a suffering of patients who have a cosmetic surgery done are often from the preparation and execution face recovery such as cutting, suture and pulling the skin of the face etc. The result of an operation, which causes changes on the face, are a direct impact on the process of face gesture pattern recognition. Thus, we recommend using the Muscle Distributed Sensor which is formed by a PANDA ring resonator circuit for face gesture pattern recognition, which will be applied in the face off recovery [4].

Several years ago, a new system of microring sensing transducer using a PANDA ring resonator type called the distributed sensors [5] was proposed. This new system has shown interesting results including the benefit of the accuracy of measurements in the nanoscale range. In recent years, the optical muscle sensing system has been presented by Yothapakdee et al. [6], in which the interesting results are the optical sensing and the self-calibration sensor base on ring resonator. This system has been designed to be used as sensors to detect the mechanism of facial muscle contractions, the strength of contraction, and degrees of perturbation of the facial muscles. Figure 1, from [6], shows the schematic diagram of the optical muscle sensing system presenting a PANDA ring resonator (1), the comparison of signals between the reference and the sensing signals in non-perturbed situation (2), and in perturbed situation (3). So, the relationships between the change in a wavelength and the perturbation of the muscle contraction can be explained through the principle of the change within the optical path length (Figure 1).

The muscle distributed sensing system has been devised and modified on the optical muscle sensing system. This particular system was based on the assumption that all of the sensors can sense and measure face gestures based on the movement or the contraction of the facial muscles used in each gesture. Therefore, the signal data with accuracy and precision is collected by using these sensing systems to store various patterns of those signals. In principle, thin film or thin plastic mask was attached to the optical muscle sensor position that has been defined, as shown in Figure 2(1) and Figure 2(2). Each of the sensors, which are placed on the face in various positions, is used to measure patterns of change after the movement of facial muscles to facial gestures. After that, the changes affecting each sensor is converted to the degree of the force through the process of the optical muscle sensing system as shown in Figure 2(3). Finally, the signal data of facial gestures obtained from measurements of the sensors is collected in order to be applied in the facial gesture pattern recognition as shown in Figure 2(4).

The muscle distributed network sensor was used to measure the various signals of facial gestures. Many details of the signal patterns of each gesture are collected by sensors distributed in an important position contributing to the analysis of these signals with accuracy and reliability. The surrounding area of the muscle expression was used as the main area for signal detection. These signals are extremely useful to medical practitioners and stakeholders who will be using them in the process of analyzing and evaluating the possibility of performing the surgery in the next step. In terms of security, this information can be

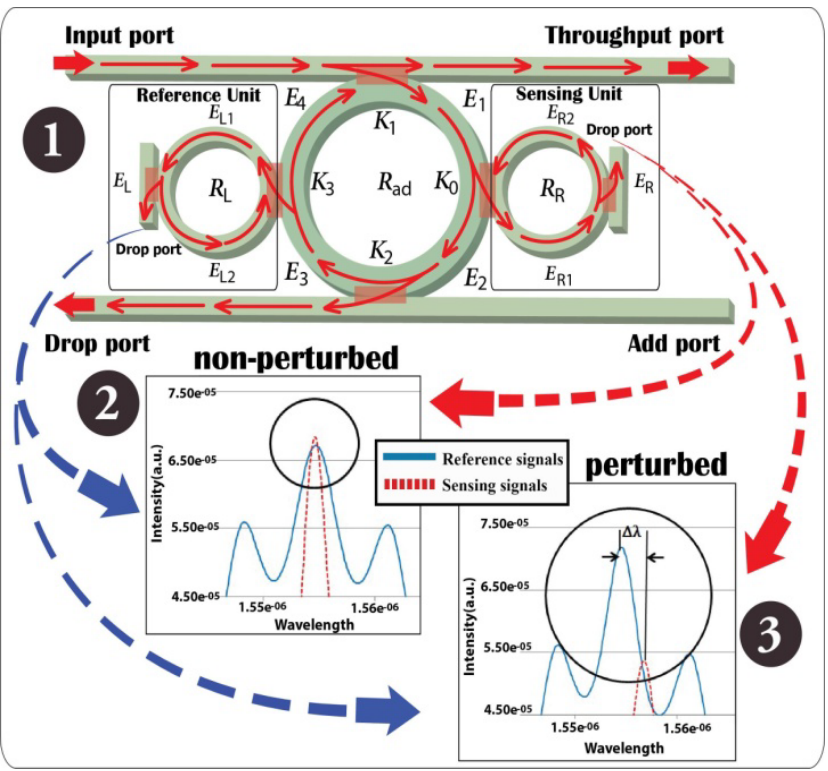

Figure 1: The schematic diagram of the optical muscle sensing system.

*Corresponding author: Yupapin P, Faculty of Electrical and Electronics Engineering, Ton Duc Thang University, Ho Chi Minh City, Vietnam, Tel: 84837755028; E-mail: preecha.yupapin@tdt.edu.vn

Received August 29, 2016; Accepted August 30, 2016; Published September 10, 2016

Citation: Yothapakdee K, Yupapin P (2016) Face Gesture Pattern Recognition using Muscle Distributed Sensor for Face off Recovery. J Biosens Bioelectron 7: e144. doi:10.4172/2155-6210.1000e144

Copyright: @ 2016 Yothapakdee K, et al. This is an open-access article distributed under the terms of the Creative Commons Attribution License, which permits unrestricted use, distribution, and reproduction in any medium, provided the original author and source are credited. 
Citation: Yothapakdee K, Yupapin P (2016) Face Gesture Pattern Recognition using Muscle Distributed Sensor for Face off Recovery. J Biosens Bioelectron 7: e144. doi:10.4172/2155-6210.1000e144

Page 2 of 2

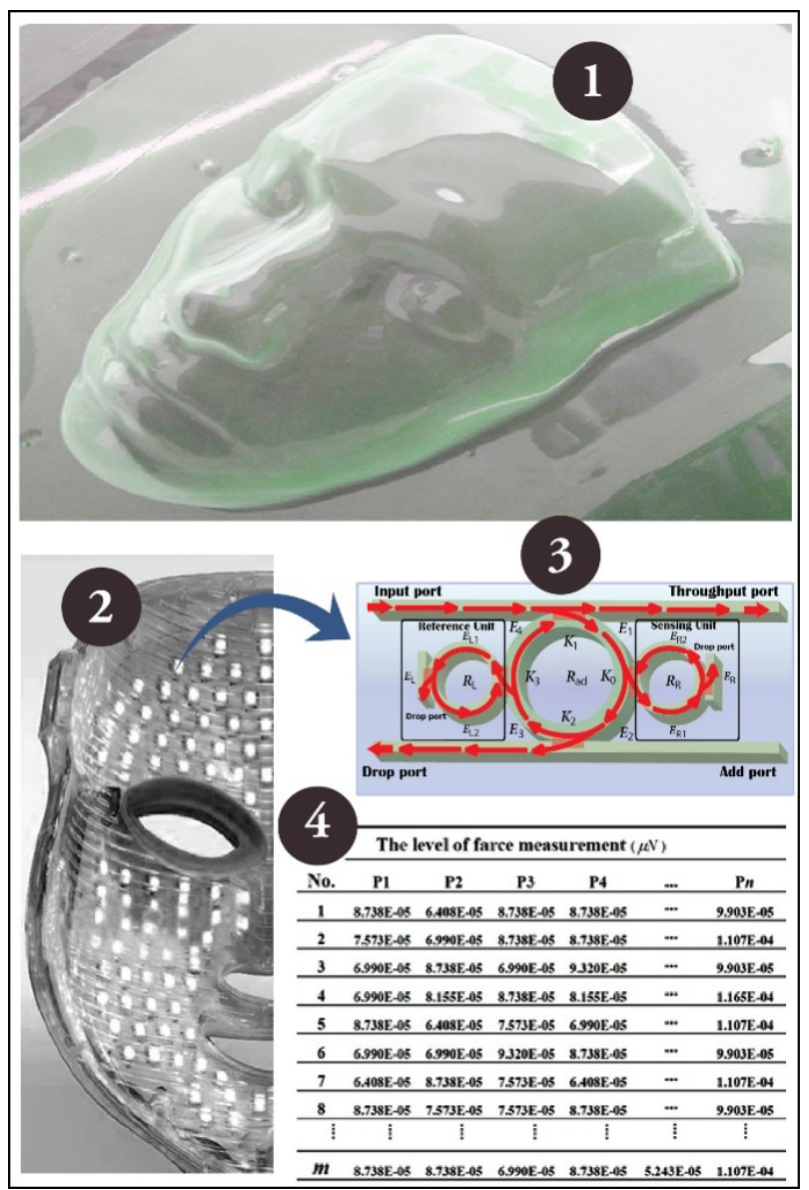

Figure 2: The proposed design muscle distributed sensor system presenting (1) the front of the mask, (2) the inside of the mask and distributed sensor, (3) the optical muscle sensing system, and (4) the level of force measurement. used to compare the biological information of the face, which is very useful for the process or mechanism to monitor the changing face of a criminal. This is an important data to be applied to research and can be crucial in resolving issues along with various assistance systems such as human-robot interaction (HRI), human computer interaction (HCI), and human machine interaction (HMI) in the near future (Figure 2).

\section{References}

1. Chou JK, Yang CK, Gong SD (2012) Face-off: Automatic Alteration of Facial Features. Multimed Tools Appl 56: 569-596.

2. Park DM (2015) Total Facelift: Forehead Lift, Midface Lift, and Neck Lift. Archives of Plastic Surgery 42: 111-125.

3. Jaafar A, Chornenky VI (2005) Apparatus and Method for Reducing Subcutaneous Fat Deposits, Virtual Face Lift and Body Sculpturing by Electroporation. US 6892099 B2.

4. Uomwech K, Sarapat K, Yupapin PP (2010) Dynamic Modulated Gaussian Pulse Propagation within the Double PANDA Ring Resonator System. Microw Opt Technol Lett 521: 1818-1821.

5. Tamee K, Srinuanjan K, Mitatha S, Yupapin PP (2011) Distributed Sensors Using a PANDA Ring Resonator Type in Multiwavelength Router. IEEE Sensors Journal 11: 1987-1992.

6. Yothapakdee K, Yupapin PP, Tamee K (2016) Facial Gesture Measuremen using Optical Muscle Sensing System. Nano Biomed Eng 4: 169-179. 